\title{
Ground State Entropy of Potts Antiferromagnets and the Approach to the 2D Thermodynamic Limit
}

\author{
Robert Shrock* , Shan-Ho Tsai ${ }^{\dagger}$ \\ Institute for Theoretical Physics \\ State University of New York \\ Stony Brook, N. Y. 11794-3840
}

\begin{abstract}
We study the ground state degeneracy per site (exponent of the ground state entropy) $W\left(\Lambda,\left(L_{x}=\infty\right) \times L_{y}, q\right)$ for the $q$-state Potts antiferromagnet on infinitely long strips with width $L_{y}$ of $2 \mathrm{D}$ lattices $\Lambda$ with free and periodic boundary conditions in the $y$ direction, denoted $\mathrm{FBC}_{y}$ and $\mathrm{PBC}_{y}$. We show that the approach of $W$ to its $2 \mathrm{D}$ thermodynamic limit as $L_{y}$ increases is quite rapid; for moderate values of $q$ and $L_{y} \simeq 4, W\left(\Lambda,\left(L_{x}=\infty\right) \times L_{y}, q\right)$ is within about $5 \%$ and $\mathcal{O}\left(10^{-3}\right)$ of the $2 \mathrm{D}$ value $W\left(\Lambda,\left(L_{x}=\infty\right) \times\left(L_{y}=\infty\right), q\right)$ for $\mathrm{FBC}_{y}$ and $\mathrm{PBC}_{y}$, respectively. The approach of $W$ to the $2 \mathrm{D}$ thermodynamic limit is proved to be monotonic (non-monotonic) for $\mathrm{FBC}_{y}\left(\mathrm{PBC}_{y}\right)$. It is noted that ground state entropy determinations on infinite strips can be used to obtain the central charge for cases with critical ground states.

05.20.-y, 64.60.C, 75.10.H
\end{abstract}

*email: shrock@insti.physics.sunysb.edu

†email: tsai@insti.physics.sunysb.edu 


\section{INTRODUCTION}

Nonzero ground state entropy, $S_{0} \neq 0$, is an important subject in statistical mechanics. One physical example is provided by ice, for which the residual molar entropy is $S_{0}=$ $0.82 \pm 0.05 \mathrm{cal} /\left(\mathrm{K}\right.$-mole), i.e., $S_{0} / R=0.41 \pm 0.03$, where $R=N_{A v o g} k_{B}$ [1].2]. This is equivalent to a ground state degeneracy per site $W>1$, since $S_{0}=k_{B} \ln W$. Such nonzero ground state entropy violates the third law of thermodynamics (see, e.g., [3, [4). The $q$-state Potts antiferromagnet (AF) [5,6] exhibits nonzero ground state entropy (without frustration) for sufficiently large $q$ on a given lattice $\Lambda$ and serves as a useful model for the study of this phenomenon. There is an interesting connection with graph theory here, since the zerotemperature partition function of the above-mentioned $q$-state Potts antiferromagnet on a graph $G$ satisfies $Z(G, q, T=0)_{P A F}=P(G, q)$, where $P(G, q)$ is the chromatic polynomial expressing the number of ways of coloring the vertices of the graph $G$ with $q$ colors such that no two adjacent vertices have the same color [7]. Thus,

$$
W\left(\left[\lim _{n \rightarrow \infty} G\right], q\right)=\lim _{n \rightarrow \infty} P(G, q)^{1 / n}
$$

where $n=v(G)$ is the number of vertices of $G$. Nontrivial exact solutions for this function are known in only a very few cases, all for 2D lattices: the square lattice for $q=3$ [8], triangular lattice and, for $q=3$, the kagomé lattice [9]. Of course, one can use large $-q$ series expansions [10 14], rigorous upper and lower bounds 15, 12 14, and Monte Carlo simulations (see, e.g., [16] [1] ). It is also worthwhile to generalize $q$ from $\mathbb{Z}_{+}$to $\mathbb{C}$ and study $W(\{G\}, q)$ in the complex $q$ plane for infinite- $n$ limits of various families of graphs, $\{G\}$ [19][26. On the positive real axis, $W(\{G\}, q)$ is an analytic function down to a point which we denote $q_{c}(\{G\})$ 22].

Since it is possible to obtain exact analytic solutions for infinitely long strips of 2D lattices [25], one has an alternate way to investigate $W(\Lambda, q)$ for $2 \mathrm{D}$ lattices, namely to calculate exactly $W$ on such infinitely long strips of progressively greater widths. These $W$ functions for infinitely long strips have interesting analytic structure in their own right, which was investigated in detail in Ref. [25]. Here we shall use them for a different purpose: to investigate how rapidly the $2 \mathrm{D}$ thermodynamic limit is approached as the width of the strips increases. We find that this approach is quite rapid. Of course, to demonstrate this does not require the use of exact analytic results; it can be seen equivalently from numerical Monte Carlo measurements on rectangular $L_{x} \times L_{y}$ patches after an extrapolation to $L_{x}=\infty$. Indeed, Monte Carlo measurements would be the standard method for this purpose since they are not limited, as the exact analytic calculations are, to a rather small range of $L_{y}$ values. However, the value of discussing this with exact results is that the reader can verify 
the conclusions directly rather than having to reproduce them with another Monte Carlo study.

The organization of the paper is as follows. In section 2 we discuss some generalities of our approach. In section 3 we prove that if one uses free (periodic) boundary conditions in the $y$ direction transverse to the length of the infinite strip, then $W$ approaches its $2 \mathrm{D}$ thermodynamic limit monotonically (nonmonotonically). Section 4 contains the numerical results for strips of the square, triangular, and honeycomb lattices with free transverse boundary conditions, while section 5 contains analogous results for periodic transverse boundary conditions. In section 6 we remark on how these strip studies can be used to determine the central charge for cases with critical ground states. Our conclusions are presented in section 7.

\section{II. $W$ ON STRIP GRAPHS AND THE APPROACH TO 2D THERMODYNAMIC LIMIT}

The usual thermodynamic limit of the Potts antiferromagnet or other statistical mechanical model on the 2D lattice $\Lambda$ involves taking $L_{x} \rightarrow \infty$ and $L_{y} \rightarrow \infty$ with fixed $L_{y} / L_{x}=\rho$, where $(\rho \neq 0, \infty)$. The question of how various thermodynamic quantities approach their 2D limits as a function of $\rho$ has been of interest for many years (e.g., Ref. 27] for the Ising model). As noted in the introduction, a different way to approach the $2 \mathrm{D}$ thermodynamic limit is via a sequence of infinitely long strips of progressively greater and greater widths. That this is different is clear from the fact that for each such strip, regardless of how large $L_{y}$ is, the ratio $L_{y} / L_{x}=0$. We picture the strip graphs as extending longitudinally in the horizontal $(x)$ direction and having a width of $L_{y}$ vertices in the vertical direction. A priori, it is not clear that this different approach will yield results that are useful to the study of the 2D thermodynamic limit, because, for a given thermodynamic quantity of interest, these results might be dominated by the fundamentally 1D nature of the infinite strip. Indeed, to illustrate a case where it is not useful, consider a model, such as a discrete ferromagnet, which has a second-order phase transition at some critical temperature $T_{c}(\Lambda)$ on a $2 \mathrm{D}$ lattice $\Lambda$, and assume that there is no exact solution of this model. If one were to try to employ exact solutions of the model on infinitely long strips of lattice type $\Lambda$ to determine $T_{c}(\Lambda)$ for the $2 \mathrm{D}$ lattice, one would get the $1 \mathrm{D}$ result $T_{c}=0$ for any finite value of $L_{y}$. Hence, for $T_{c}(\Lambda)$ this method would not give any useful information. However, as we shall show, the situation is very different with the ground state entropy $S_{0}(\Lambda)$; for this quantity, one can use results on infinite strips to get quite accurate values even for rather modest strip widths. 
The existence of the thermodynamic limit for the 2D lattice $\Lambda$ means that the maximal finite real $q$ where $W(\Lambda, q)$ is nonanalytic, $q_{c}(\Lambda)$, is independent of the boundary conditions used in taking the 2D thermodynamic limit [22]. Let us denote the $W$ function for the $L_{x} \times L_{y}$ strip of the lattice of type $\Lambda$ as $W\left(\Lambda\left(L_{x} \times L_{y}\right), B C_{x}, B C_{y}, q\right)$. We observe here that for physical (positive integral) $q>q_{c}(\Lambda)$, in the limit $L_{x} \rightarrow \infty$, this $W$ function is independent of the boundary conditions used in the $x$ direction. This is also true for real $q>q_{c}(\Lambda)$ (and more generally, in the region of the complex $q$ plane denoted $R_{1}$ in our previous studies [22]). We thus introduce a more compact notation for the $W$ function on infinitely long strips:

$$
W\left(\Lambda\left(L_{y}\right), B C_{y}, q\right) \equiv \lim _{L_{x} \rightarrow \infty} W\left(\Lambda\left(L_{x} \times L_{y}\right), B C_{x}, B C_{y}, q\right)
$$

Indeed, let $\Lambda_{d}$ be an infinite $d$-dimensional lattice and $\Lambda_{d-1, L_{d}}$ be a slab of a $(d-1)$ dimensional lattice, infinite in $d-1$ dimensions and of finite thickness $L_{d}$ in the $d$ 'th dimension. For physical $q>q_{c}\left(\Lambda_{d}\right)$, the value of $W\left(\Lambda_{d-1, L_{d}}, q\right)$ is independent of the boundary conditions used for the $(d-1)$ directions when taking $L_{j} \rightarrow \infty$ for $1 \leq j \leq d-1$. As we have discussed before [22], this is not true for all $q \in \mathbb{C}$; however, here we deal only with physical $q$ values.

\section{ISSUE OF MONOTONICITY OF APPROACH TO 2D THERMODYNAMIC LIMIT}

In this section we show that for free (periodic) boundary conditions in the $y$ direction, $W$ for infinitely long strips of width $L_{y}$ approaches its 2D thermodynamic limit monotonically (nonmonotonically) as $L_{y} \rightarrow \infty$.

\section{A. Monotonic Approach for $\mathbf{F B C}_{y}$}

We begin with the case of free boundary conditions and state the following theorem:

Theorem 1

Let $\Lambda_{d-1, L_{d}}$ denote a regular lattice graph of infinite extent in $d-1$ dimensions and width (thickness) $L_{d}$ in the $d$ 'th dimension. Let the boundary conditions in the $d$ 'th direction be free and the boundary conditions in each of the first $d-1$ be (separately) free or periodic. 
(Note that $\Lambda_{d-1,1} \equiv \Lambda_{d-1}$ and $\Lambda_{d-1, \infty}=\Lambda_{d}$ ) Then for fixed $q>q_{c}\left(\Lambda_{d}\right), W\left(\Lambda_{d-1, L_{d}}, q\right)$ is a monotonically decreasing function of $L_{d}$ for $1 \leq L_{d} \leq \infty$.

Proof

We shall prove the theorem for the case $d=2$; its generalization to $d \geq 3$ will be obvious. We consider a finite strip graph of the lattice, of size $L_{x} \times L_{y}$, where the longitudinal direction is $x$. Assume that one has made an allowed coloring of this graph. Now connect another layer of sites to the layer that formerly constituted the top layer of sites on the strip. The coloring of this new layer of sites imposes additional constraints on the coloring of the original strip, and excludes a certain subset of what were previously allowed colorings. Thus, the fraction of sites on the augmented graph that have more constraints increases; i.e., the sites on the upper and lower edges, which have fewer constraints on their coloring because of the free transverse boundary conditions, constitute a progressively smaller fraction of the total number of sites as $L_{y}$ increases. Hence, the chromatic polynomial per site, $P\left(\Lambda,\left[L_{x} \times L_{y}\right], q\right)^{1 / n}$, decreases. This inequality holds for arbitrary $L_{x}$. Taking the limit as $L_{x} \rightarrow \infty$ and using the definition (1.1), one obtains the theorem for the case $d=2$. A straightforward generalization of this argument proves the theorem for $d \geq 3$.

A corollary of this theorem is that if one compares $W$ on two infinite lattices of the same type and of different dimensions, such as $d$-dimensional cartesian lattices then, for fixed $q>q_{c}(\Lambda)$

$$
W\left(\Lambda_{d}, q\right)<W\left(\Lambda_{d^{\prime}}, q\right) \quad \text { if } \quad d>d^{\prime}
$$

To prove this corollary, one starts with $d^{\prime}=d-1$ and (i) constructs $\Lambda_{d}$ from $\Lambda_{d-1}$ by imposing free boundary conditions in the $d^{\prime}$ 'th direction and adding layers of vertices in this $d$ 'th direction, (ii) uses the monotonicity relation of theorem 1 for the quantities $W\left(\Lambda_{d-1, L_{d}}, q\right)$, and (iii) takes the number of added layers in the $d^{\prime}$ th direction to infinity to get $\Lambda_{d}$. The monotonicity relation for the infinite lattices (3.1) was previously noted by Chow and $\mathrm{Wu}$ [4]. It is important to observe that the monotonicity relation (3.1) does not imply our monotonicity theorem 1. This is clear from the fact that the inequality (3.1) holds independent of the boundary conditions that one uses to define the respective thermodynamic limits on $\Lambda_{d}$ and $\Lambda_{d-1}$, whereas, on the contrary, the inequality in our theorem 1 does not apply if one uses periodic boundary conditions for the $d$ 'th direction of the $(d-1)$-dimensional strip or slab of width $L_{d}$ (see below). 


\section{B. Non-monotonic Approach for $\mathbf{P B C}_{y}$}

Next, we show that a similar monotonicity result does not hold if one imposes periodic boundary conditions in the $d$ 'th direction. This is clear from the proof, since the greater freedom in coloring the sites on the boundary layer in the $d$ 'th dimension played a crucial role, but if one imposes periodic boundary conditions in the d'th direction, there is no such boundary layer. The simplest illustration is provided by the case $d=1$, for which [22] $q_{c}\left(\Lambda_{1}\right)=2$ and

$$
W\left(\Lambda_{1}, q\right)=q-1
$$

For free boundary conditions, the function that enters on the right-hand side of eq. (1.1) is

$$
P\left(\left(\Lambda_{1}\right)_{n}, F B C, q\right)^{1 / n}=q^{1 / n}(q-1)^{1-\frac{1}{n}}
$$

For fixed $q \geq q_{c}\left(\Lambda_{1}\right)$, this is a monotonically decreasing function of $n$ as $n$ increases from 1 to infinity. However, if we impose periodic boundary conditions, i.e. deal with an $n$-vertex circuit graph $C_{n}$ 円, then, the function that enters on the right-hand side of eq. (1.1) is

$$
P\left(\left(\Lambda_{1}\right)_{n}, P B C, q\right)^{1 / n}=(q-1)\left[1+(-1)^{n}(q-1)^{-(n-1)}\right]^{1 / n}
$$

This is a non-monotonic function of $n$. For example, for the lowest value of $q$ where the 1D Potts AF has nonzero ground state entropy, viz., $q=3$, for which the $n \rightarrow \infty$ limit is $W\left(\Lambda_{1}, q=3\right)=2$, eq. (3.4) exhibits the non-monotonic behavior indicated by the values $6^{1 / 3}=1.817$. for $n=3,(18)^{1 / 4}=2.060$. for $n=4,(30)^{1 / 5}=1.974$. for $n=5,(66)^{1 / 6}=$ 2.010. for $n=6$, etc. Similar non-monotonic behavior occurs for higher values of $q$. Looking at subsequences, we find that $P\left(\left(\Lambda_{1}\right)_{n}, P B C, q\right)^{1 / n}$ is a monotonically increasing function of $n$ for odd $n \geq 3$ and a monotonically decreasing function of $n$ for even $n \geq 2$. This is connected with the fact that the circuit graph $\left[\left(\Lambda_{1}\right)_{n}, P B C\right]=C_{n}$ with odd (even) $n$ has chromatic number $\chi=3(\chi=2)$. The different behaviors of $P\left(\left(\Lambda_{1}\right)_{n}, F B C, q\right)^{1 / n}$ and $P\left(\left(\Lambda_{1}\right)_{n}, P B C, q\right)^{1 / n}$ can be seen in a more general context by analytically continuing eqs.

\footnotetext{
${ }^{1}$ Parenthetically, we note that $C_{n}$ is only a (proper) graph for $n \geq 3$ since the strict mathematical definition of a graph forbids (i) any multiple bond connecting a given pair of vertices (present for $C_{n=2}$ ) and (ii) any bond going out from a given vertex and looping back to the same vertex (present for $C_{n=1}$ ). This is not important for our demonstration of non-monotonicity.
} 
(3.3) and (3.4) from $n \in \mathbb{Z}_{+}$to $n \in \mathbb{R}_{+}$and plotting them as functions of $n$ (in the second case, since $P\left(C_{n}, q\right)=(q-1)^{n}+(-1)^{n}(q-1)$ is complex for $n \notin \mathbb{Z}$, we plot $\left.\left|P\left(C_{n}, q\right)\right|^{1 / n}\right)$. This is shown in Fig. 1. One notices that although eq. (3.4) for periodic boundary conditions behaves non-monotonically, it approaches the $n=\infty$ value $W\left(\Lambda_{1}, q=3\right)=2$ considerably more rapidly than the FBC expression, eq. (3.3). As one increases $q$ beyond 3, the first maximum in $\left|P\left(C_{n}, q\right)\right|^{1 / n}$ moves slightly leftward, and the oscillations damp out faster. As we shall show in the tables below, a similar difference holds between the behavior of $W\left(\Lambda\left(L_{y}\right), B C_{y}, q\right)$ for $\mathrm{FBC}_{y}$ and $\mathrm{PBC}_{y}$.

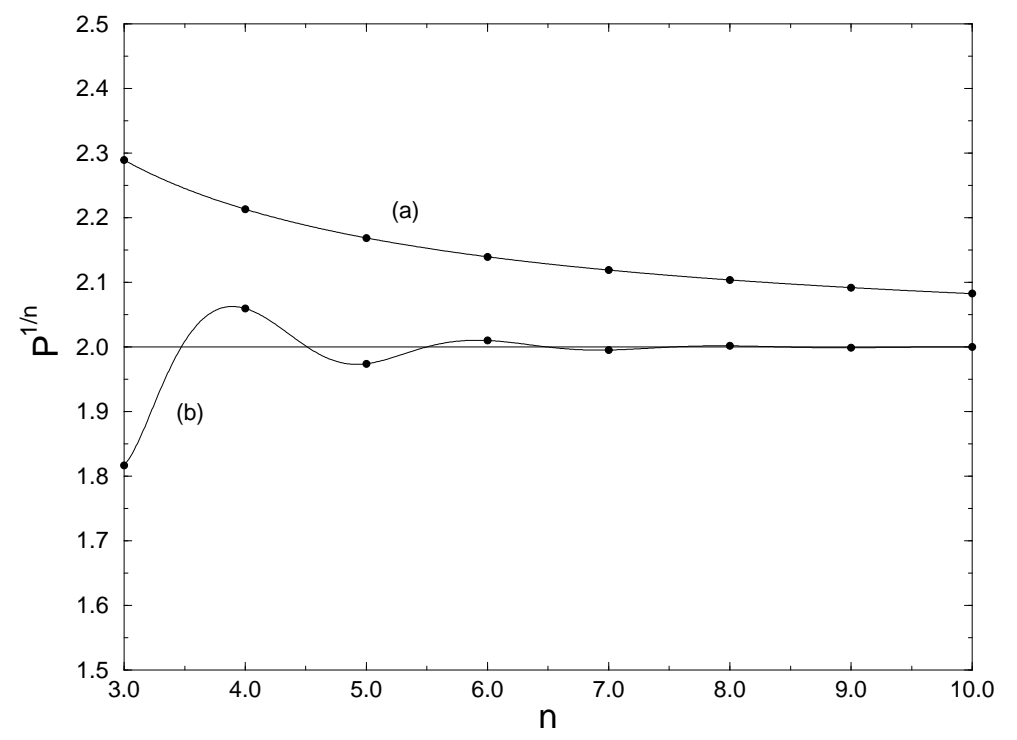

FIG. 1. Plots of (a) eq. (3.3) and (b) eq. (3.4) for $q=3$, as functions of real $n$, together with physical values for integer $n$. Horizontal line is the asymptote, $W\left(\Lambda_{1}, q=3\right)=2$.

\section{QUANTITATIVE RESULTS FOR STRIPS WITH FBC F $_{y}$}

One would like to go beyond the general inequality in Theorem 1 to obtain an explicit numerical determination of the dependence of $W$ on $L_{d}$. We do this here for $d=2$ and, in particular, for the square (sq), triangular (t), and honeycomb (hc) lattices. For the strip graph of each type $G_{s}$, we define the ratio

$$
R_{W}\left(\Lambda\left(L_{y}\right), B C_{y}, q\right)=\frac{W\left(\Lambda\left(L_{y}\right), B C_{y}, q\right)}{W(\Lambda, q)}
$$

In the appendix we list the exact analytic expressions for $W\left(\Lambda\left(L_{y}\right), F B C_{y}, q\right)$ for $\Lambda=s q, t, h c$

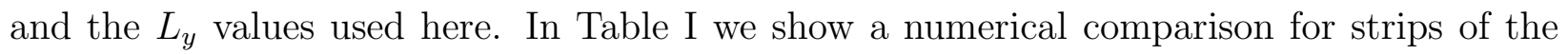


square lattice (along the row direction) for $1 \leq L_{y} \leq 4$ and $3 \leq q \leq 10$. The exact value $W(s q, q=3)=(4 / 3)^{3 / 2}=1.53960 \ldots$ is from Ref. [8], while the values of $W(s q, q)$ for $4 \leq q \leq 10$ are from our Monte Carlo measurements in Ref. [22]. Using the conservatively quoted uncertainties that we gave for the Monte Carlo measurements, it would follow that the corresponding uncertainties in the ratios (4.1) are $\sim(3-4) \times 10^{-4}$; with less conservative estimates of uncertainties in the Monte Carlo measurements, the resultant uncertainties in these ratios would be smaller. In Tables 【1 and 【II we give the analogous comparisons for strips of the triangular lattice of widths $L_{y}=2,3,4$ and of the honeycomb lattice for widths $L_{y}=2$ and 3. In all of these cases, one observes that, for fixed $q$, the agreement with the infinite-lattice value gets better as the width increases and for fixed width, the agreement gets better as the value of $q$ increases. These comparisons show that the approach to the $2 \mathrm{D}$ thermodynamic limit is reasonably rapid even for free boundary conditions in the transverse direction. For example, for an $\infty \times 4$ strip of the square lattice for $3 \leq q \leq 5$, the $W$ values are within about $5 \%$ of their respective values for the infinite 2 D lattice. 
TABLE I. Comparison of values of $W\left(s q\left(L_{y}\right), F B C_{y}, q\right)$ with $W(s q, q)$ for $3 \leq q \leq 10$. For each value of $q$, the quantities in the upper line are identified at the top and the quantities in the lower line are the values of $R_{W}\left(s q\left(L_{y}\right), F B C_{y}, q\right)$. The $\mathrm{FBC}_{y}$ is symbolized as $F$ in the table.

\begin{tabular}{|c|c|c|c|c|c|c|c|}
\hline$q$ & $W(s q(1), F, q)$ & $W(s q(2), F, q)$ & $W(s q(3), F, q)$ & $W(s q(4), F, q)$ & $W(s q, q)$ & $W(s q, q)_{s}$ & $W(s q, q)_{\ell}$ \\
\hline \multirow[t]{2}{*}{3} & 2 & 1.73205 & 1.65846 & 1.624945 & $1.53960 .$. & 1.53960. & 1.50000 \\
\hline & 1.299 & 1.125 & 1.077 & 1.055 & 1 & 1 & \\
\hline \multirow[t]{2}{*}{4} & 3 & 2.64575 & 2.53800 & 2.48590 & $2.3370(7)$ & 2.3361 & 2.33333 \\
\hline & 1.284 & 1.132 & 1.086 & 1.064 & 1 & - & - \\
\hline \multirow[t]{2}{*}{5} & 4 & 3.60555 & 3.48304 & 3.42336 & $3.2510(10)$ & 3.2504 & 3.25000 \\
\hline & 1.230 & 1.109 & 1.071 & 1.053 & 1 & - & - \\
\hline \multirow[t]{2}{*}{6} & 5 & 4.58258 & 4.45136 & 4.38717 & $4.2003(12)$ & 4.2001 & 4.20000 \\
\hline & 1.190 & 1.091 & 1.060 & 1.0445 & 1 & - & - \\
\hline \multirow[t]{2}{*}{7} & 6 & 5.56776 & 5.43073 & 5.36348 & $5.1669(15)$ & 5.1667 & 5.16667 \\
\hline & 1.161 & 1.078 & 1.051 & 1.038 & 1 & - & - \\
\hline \multirow[t]{2}{*}{8} & 7 & 6.55744 & 6.41623 & 6.34677 & $6.1431(20)$ & 6.1429 & 6.14286 \\
\hline & 1.1395 & 1.067 & 1.0445 & 1.033 & 1 & - & - \\
\hline \multirow[t]{2}{*}{9} & 8 & 7.54983 & 7.40548 & 7.33434 & $7.1254(22)$ & 7.1250 & 7.12500 \\
\hline & 1.123 & 1.060 & 1.039 & 1.029 & 1 & - & - \\
\hline \multirow[t]{2}{*}{10} & 9 & 8.54400 & 8.39720 & 8.324745 & $8.1122(25)$ & 8.1111 & 8.11111 \\
\hline & 1.109 & 1.053 & 1.035 & 1.026 & 1 & - & - \\
\hline
\end{tabular}


TABLE II. Comparison of values of $W\left(t\left(L_{y}\right), F B C_{y}, q\right)$ with $W(t, q)$ for $3 \leq q \leq 10$. For each value of $q$, the quantities in the upper line are identified at the top and the quantities in the lower line are the values of $R_{W}\left(t\left(L_{y}\right), F B C_{y}, q\right)$. The $\mathrm{FBC}_{y}$ is symbolized as $F$ in the table.

\begin{tabular}{|c|c|c|c|c|c|}
\hline$q$ & $W(t(2), F, q)$ & $W(t(3), F, q)$ & $W(t(4), F, q)$ & $W(t, q)$ & $W(t, q)_{\ell}$ \\
\hline \multirow[t]{2}{*}{4} & 2 & 1.77173 & 1.67619 & 1.46100 & 1.333333 \\
\hline & 1.369 & 1.213 & 1.147 & 1 & - \\
\hline \multirow[t]{2}{*}{5} & 3 & 2.72998 & 2.60495 & 2.26411 & 2.250000 \\
\hline & 1.325 & 1.206 & 1.151 & 1 & - \\
\hline \multirow[t]{2}{*}{6} & 4 & 3.71457 & 3.579715 & 3.20388 & 3.200000 \\
\hline & 1.248 & 1.159 & 1.117 & 1 & - \\
\hline \multirow[t]{2}{*}{7} & 5 & 4.70571 & 4.56515 & 4.16819 & 4.166667 \\
\hline & 1.200 & 1.129 & 1.095 & 1 & - \\
\hline \multirow[t]{2}{*}{8} & 6 & 5.69974 & 5.55530 & 5.14358 & 5.142857 \\
\hline & 1.167 & 1.108 & 1.080 & 1 & - \\
\hline \multirow[t]{2}{*}{9} & 7 & 6.695395 & 6.54810 & 6.12539 & 6.125000 \\
\hline & 1.143 & 1.093 & 1.069 & 1 & - \\
\hline \multirow[t]{2}{*}{10} & 8 & 7.69208 & 7.54259 & 7.11134 & 7.111111 \\
\hline & 1.125 & 1.082 & 1.061 & 1 & - \\
\hline
\end{tabular}


TABLE III. Comparison of values of $W\left(h c\left(L_{y}\right), F B C_{y}, q\right)$ with $W(h c, q)$ for $3 \leq q \leq 10$. For each value of $q$, the quantities in the upper line are identified at the top and the quantities in the lower line are the values of $R_{W}\left(h c\left(L_{y}\right), F B C_{y}, q\right)$. The $\mathrm{FBC}_{y}$ is symbolized as $F$ in the table.

\begin{tabular}{|c|c|c|c|c|c|}
\hline$q$ & $W(h c(2), F, q)$ & $W(h c(3), F, q)$ & $W(h c, q)$ & $W(h c, q)_{\text {ser. }}$ & $W(h c, q)_{\ell}$ \\
\hline \multirow[t]{2}{*}{3} & 1.82116 & 1.76567 & $1.6600(5)$ & 1.6600 & 1.658312 \\
\hline & 1.097 & 1.064 & 1 & - & - \\
\hline \multirow[t]{2}{*}{4} & 2.79468 & 2.72942 & $2.6038(7)$ & 2.6034 & 2.603417 \\
\hline & 1.073 & 1.048 & 1 & - & - \\
\hline \multirow[t]{2}{*}{5} & 3.78389 & 3.71448 & $3.5796(10)$ & 3.5795 & 3.579455 \\
\hline & 1.057 & 1.038 & 1 & - & - \\
\hline \multirow[t]{2}{*}{6} & 4.77760 & 4.70568 & $4.5654(15)$ & 4.5651 & 4.565085 \\
\hline & 1.046 & 1.031 & 1 & - & - \\
\hline \multirow[t]{2}{*}{7} & 5.77336 & 5.69973 & $5.5556(17)$ & 5.5553 & 5.555278 \\
\hline & 1.039 & 1.026 & 1 & - & - \\
\hline \multirow[t]{2}{*}{8} & 6.77028 & 6.69539 & $6.5479(20)$ & 6.5481 & 6.548095 \\
\hline & 1.034 & 1.023 & 1 & - & - \\
\hline \multirow[t]{2}{*}{9} & 7.76793 & 7.69208 & $7.5424(22)$ & 7.5426 & 7.542587 \\
\hline & 1.030 & 1.020 & 1 & - & - \\
\hline \multirow[t]{2}{*}{10} & 8.76607 & 8.68945 & $8.5386(25)$ & 8.5382 & 8.538222 \\
\hline & 1.027 & 1.018 & 1 & - & - \\
\hline
\end{tabular}




\section{QUANTITATIVE RESULTS FOR STRIPS WITH PBC ${ }_{y}$}

In Tables $[\nabla$ and $\nabla$ we present similar results for infinite strips with periodic boundary conditions in the transverse $(y)$ direction. The exact analytic expressions that we use for these tables are given in the Appendix. As is mentioned in the Appendix, for a strip of the square lattice with $\mathrm{PBC}_{y}$ and cross sections forming triangles, depending on one's labelling conventions, this corresponds to $L_{y}=3$ or $L_{y}=4$, where in the latter case, one interprets the periodic boundary conditions as identifying the top and bottom vertices for each value of $x$. A similar comment applies for a strip with $\mathrm{PBC}_{y}$ and transverse cross sections forming squares. For the table, we use the convention of choosing the smaller of the respective values of $L_{y}$. We find that for a given $q, W$ approaches its $2 \mathrm{D}$ value $W(\Lambda, q)$ much more rapidly with periodic rather than free transverse boundary conditions: for the modest width $L_{y}=4$, $W$ is within $\mathcal{O}\left(10^{-3}\right)$ of its $2 \mathrm{D}$ value for moderate $q$. The finding that the periodic boundary conditions in the transverse direction yield a more rapid approach to the $2 \mathrm{D}$ thermodynamic limit than the free boundary conditions is not, in itself, a surprise; this is in accord with a wealth of past experience with statistical mechanical models on finite-size lattices. What is remarkable is how rapid in absolute terms this approach is. Of course, one can also consider larger values of $L_{y}$, but the strikingly rapid approach to the $2 \mathrm{D}$ thermodynamic limit is already fully demonstrated by the range of $L_{y}$ that we have considered.

TABLE IV. Comparison of values of $W\left(s q\left(L_{y}\right), P B C_{y}, q\right)$ with $W(s q, q)$ for $3 \leq q \leq 10$. For each value of $q$, the quantities in the upper line are identified at the top and the quantities in the lower line are the values of $R_{W}\left(s q\left(L_{y}\right), P B C_{y}, q\right)$. The $\mathrm{PBC}_{y}$ is symbolized as $P$ in the table.

\begin{tabular}{cccc}
\hline \hline$q$ & $W(s q(3), P, q)$ & $W(s q(4), P, q)$ & $W(s q, P, q)$ \\
3 & 1.25992 & 1.58882 & $1.53960 .$. \\
& 0.8183 & 1.032 & 1 \\
4 & 2.22398 & 2.37276 & $2.3370(7)$ \\
& 0.9516 & 1.015 & 1 \\
5 & 3.17480 & 3.26878 & $3.2510(10)$ \\
& 0.9766 & 1.0055 & 1 \\
& 4.14082 & 4.21082 & $4.2003(12)$ \\
& 0.9858 & 1.002505 & 1
\end{tabular}




\begin{tabular}{lccc}
7 & 5.11723 & 5.17377 & $5.1669(15)$ \\
8 & 0.9904 & 1.0013 & 1 \\
& 6.10017 & 6.14792 & $6.1431(20)$ \\
9 & 0.9930 & 1.0008 & 1 \\
& 7.08734 & 7.12881 & 1 \\
10 & 0.9947 & 1.0005 & $8.1254(22)$ \\
& 8.07737 & 8.11409 & 1 \\
\hline \hline
\end{tabular}


TABLE V. Comparison of values of $W\left(t\left(L_{y}\right), P B C_{y}, q\right)$ with $W(t, q)$ for $4 \leq q \leq 10$. For each value of $q$, the quantities in the upper line are identified at the top and the quantities in the lower line are the values of $R_{W}\left(t\left(L_{y}\right), P B C_{y}, q\right)$. The $\mathrm{PBC}_{y}$ is symbolized as $P$ in the table.

\begin{tabular}{lccc}
\hline \hline$q$ & $W(t(3), P, q)$ & $W(t(4), P, q)$ & $W(t, q)$ \\
4 & 1.58740 & 1.18921 & 1.46100 \\
& 1.0865 & 0.8140 & 1 \\
5 & 2.35133 & 2.21336 & 2.26411 \\
& 1.0385 & 0.9776 & 1 \\
6 & 3.23961 & 3.185055 & 3.20388 \\
& 1.0112 & 0.9941 & 1 \\
& 4.17934 & 4.15965 & 4.16819 \\
7 & 1.0027 & 0.99795 & 1 \\
& 5.14256 & 5.13936 & 1 \\
8 & 0.99980 & 0.9992 & 1 \\
& 6.11803 & 6.12324 & 1 \\
9 & 0.99880 & 0.99965 & 7.11134 \\
& 7.10059 & 7.11027 & 0.12539 \\
\hline \hline & 0.99849 & 0.99985 & 1
\end{tabular}




\section{CASES WITH CRITICAL GROUND STATES}

For certain 2D lattices $\Lambda$ and values of $q$, the $q$-state Potts antiferromagnet has a critical ground state, i.e., as $T \rightarrow 0$, a correlation length $\xi$ defined, say, by a spin-spin correlation function, goes to infinity. Normally, in statistical mechanics, for a given dimensionality $d$ and symmetry group $G$, second-order phase transitions can be described by a universality class representing a fixed point of the renormalization group. Conformal field theory methods have provided a powerful way to understand these universality classes and the associated critical exponents in terms of Virasoro algebras with given central charges and scaling dimensions [28]. In addition to phase transitions involving ferromagnetic long range order at low temperatures, this is also true of antiferromagnetic transitions on bipartite lattices, but the situation is more complicated on nonbipartite lattices, as is illustrated by the fact that the isotropic Ising antiferromagnet on the triangular lattice has no finite-temperature phase transition but is critical at $T=0$.

The $q=3$ Potts antiferromagnet on the square lattice has a critical ground state with central charge $c=1$, as a consequence of the fact that at $T=0$ this model can be mapped to a critical six-vertex model [8]. From the exact solution in Ref. [9], it can be argued that the $q=4$ Potts antiferromagnet on the triangular lattice is also critical, which is closely related to the fact that the $q=3$ Potts antiferromagnet on the kagomé lattice is critical at $T=0$ 29. We recall that given the Virasoro algebra with central extension

$$
\left[L_{m}, L_{n}\right]=(m-n) L_{m+n}+\frac{c}{12} m\left(m^{2}-1\right) \delta_{m+n, 0}
$$

and the corresponding Kac-Moody algebra realized at level $k$

$$
\left[J_{m}^{a}, J_{n}^{b}\right]=c^{a b c} J_{m+n}^{c}+\frac{1}{2} k n \delta^{a b} \delta_{m+n, 0}
$$

with structure constants $c_{a b c}$, as connected via the Sugawara relation (e.g., [28)

$$
L_{n}=-\frac{1}{C_{2}(g)+k} \sum_{m=-\infty}^{\infty}: J_{m}^{a} J_{n-m}^{a}:
$$

it follows that

$$
c=\frac{\operatorname{dim}(g)}{C_{2}(g) / k+1}
$$

where $C_{2}(g)$ is the quadratic Casimir operator for the algebra $g$. In particular,

$$
g=s u(M)_{k=1} \quad \Longrightarrow \quad c=M-1
$$


Hence, from eq. (6.5) together with the finding [29] that the Kac-Moody algebra is $s u(3)_{k=1}$ for the $T=0 q=3$ Potts AF on the kagomé lattice [29], it follows that $c=2$ for this critical ground state. Given that there is a close connection between the Potts antiferromagnets with $q=3$ on the kagomé lattice and with $q=4$ on the triangular lattice, which leads to the relation $W(k a g, q=3)=W(\operatorname{tri}, q=4)^{1 / 3}[9]$, this suggests that this value of $c=2$ also holds for the $T=0, q=4$ Potts AF on the triangular lattice.

Here we would like to point out that determinations of the ground state entropy on infinitely long strips of finite width can be used to obtain the central charge $c$ for Potts antiferromagnets with critical ground states. If one considers the model on a lattice of size $L_{x} \times L_{y}$, then, in the limit as $L_{x} \rightarrow \infty$, one has [31]

$$
f_{\text {strip }, L_{y}}=f_{\text {bulk }}+\frac{f_{\text {surf } f}}{L_{y}}+\frac{\Delta}{L_{y}^{2}}+O\left(L_{y}^{-3}\right)
$$

where $f_{\text {surf. }}=0$ is nonzero (zero) for free (periodic) boundary conditions in the $y$ direction and

$$
\Delta=\left\{\begin{array}{l}
\frac{\pi}{6} c \text { for } \mathrm{PBC}_{y} \\
\frac{\pi}{24} c \text { for } \mathrm{FBC}_{y}
\end{array}\right.
$$

For the critical ground states of interest here, viz., $q=3,4,3$ on the square, triangular, and kagomé lattices, respectively, as well as other possible 2D cases, the Potts antiferromagnet exhibits ground state entropy without frustration, and the reduced free energy (per site) $f=$ $\lim _{N \rightarrow \infty} N^{-1} \ln Z$ is given simply by the ground state entropy: $f(\Lambda, q)_{P A F}=S_{0}(\Lambda, q)_{P A F} / k_{B}$, Hence, eq. (6.6) becomes

$$
S_{\text {strip }, L_{y}}=S_{\text {bulk }}+\frac{S_{\text {surf. }}}{L_{y}}+\frac{\Delta}{L_{y}^{2}}+O\left(L_{y}^{-3}\right)
$$

Thus, calculations of $S_{s t r i p, L_{y}}$ for several different values of $L_{y}$ can yield $c$. Normally, one would do this via the most general and robust method, namely Monte Carlo simulations. For small $L_{y}$ values, we have shown that it is actually possible to get exact analytic results, but this method is not competitive with Monte Carlo simulations for strips with larger values of $L_{y}$. One might note in passing that for the $q=3$ Potts AF on the infinite square strip with $\mathrm{PBC}_{y}$ and cross sections forming squares, taking $L_{y}=4$, using the fact that $S_{\text {surf. }}=0$ in this case, and dropping terms of order $L_{y}^{-3}$ in eq. (6.8), we obtain the estimate $c=0.96$, quite close to the exact value $c=1$. 


\section{CONCLUSIONS}

In summary, we have studied a different type of approach to the $2 \mathrm{D}$ thermodynamic limit for the ground state entropy, or equivalently, the ground state degeneracy per site, $W$, of the $q$-state Potts antiferromagnet, using infinitely long strips of increasing widths. We have found that the approach of $W$ to its 2D thermodynamic limit is quite rapid; for moderate values of $q$ and widths $L_{y} \simeq 4, W\left(\Lambda_{L_{y}}, q\right)$ is within about $5 \%$ and $\mathcal{O}\left(10^{-3}\right)$ of the $2 \mathrm{D}$ value for free and periodic boundary conditions, respectively. We have also proved that the approach of $W$ to the 2D thermodynamic limit is monotonic (non-monotonic) for free (periodic) boundary conditions in the transverse direction. Finally, we have noted that these ground state entropy determinations on infinite strips can be used to obtain the central charge for cases with critical ground states.

We are grateful to Prof. M. Roček for the collaborative work on Ref. [25]. This research was supported in part by the NSF grant PHY-97-22101.

\section{APPENDIX}

We gather together here the exact analytic formulas on which our numerical tables are based. It should be emphasized that the entries in these tables and the resultant conclusions about the rapidity of the approach of $W$ to the 2D thermodynamic limit for infinitely long strips with free or periodic transverse boundary conditions could also have been obtained using purely numerical Monte Carlo calculations. The usefulness of the analytic formulas (which are elementary for $L_{y}=1,2$ ) is just that they enable one to check the results more directly.

\section{A. Square Lattice, $\mathbf{F B C}_{y}$}

For infinitely long strips of the square lattice with $\mathrm{FBC}_{y}$, we have

$$
\begin{gathered}
W\left(s q\left(L_{y}=1\right), F B C_{y}, q\right)=q-1 \\
W\left(s q\left(L_{y}=2\right), F B C_{y}, q\right)=\left(q^{2}-3 q+3\right)^{1 / 2}
\end{gathered}
$$




$$
\begin{gathered}
W\left(s q\left(L_{y}=3\right), F B C_{y}, q\right)=2^{-1 / 3}\left[(q-2)\left(q^{2}-3 q+5\right)\right. \\
\left.\quad+\left[\left(q^{2}-5 q+7\right)\left(q^{4}-5 q^{3}+11 q^{2}-12 q+8\right)\right]^{1 / 2}\right]^{1 / 3}
\end{gathered}
$$

$W\left(s q\left(L_{y}=4\right), F B C_{y}, q\right)$ is given by the maximal root of the cubic equation

$$
\xi^{3}+b_{s q(4), 1} \xi^{2}+b_{s q(4), 2} \xi+b_{s q(4), 3}=0
$$

where the coefficients $b_{s q(4), k}, k=1,2,3$ were listed in Ref. [25].

\section{B. Triangular Lattice, $\mathbf{F B C}_{y}$}

For the triangular lattice strips with $\mathrm{FBC}_{y}$, we have

$$
\begin{gathered}
W\left(t\left(L_{y}=2\right), F B C_{y}, q\right)=q-2 \\
W\left(t\left(L_{y}=3\right), F B C_{y}, q\right)=2^{-1 / 3}\left[\left(q^{3}-7 q^{2}+18 q-17\right)\right. \\
\left.+\left[q^{6}-14 q^{5}+81 q^{4}-250 q^{3}+442 q^{2}-436 q+193\right]^{1 / 2}\right]^{1 / 3}
\end{gathered}
$$

$\left.W\left(t\left(L_{y}=4\right), F B C_{y}\right], q\right)$ is given by the maximal root of the quartic equation

$$
\xi^{4}+b_{t(4), 1} \xi^{3}+b_{t(4), 2} \xi^{2}+b_{t(4), 3} \xi+b_{t(4), 4}=0
$$

where the $b_{t(4), k}, k=1, \ldots, 4$ were listed in Ref. 225].

\section{Honeycomb Lattice, $\mathbf{F B C}_{y}$}

For the honeycomb lattice strips with $\mathrm{FBC}_{y}$, we have

$$
W\left(h c\left(L_{y}=2\right), F B C_{y}, q\right)=\left(q^{4}-5 q^{3}+10 q^{2}-10 q+5\right)^{1 / 4}
$$

$W\left(h c\left(L_{y}=3\right), F B C_{y}, q\right)$ is given by the maximal root of the cubic equation

$$
\xi^{3}+b_{h c(3), 1} \xi^{2}+b_{h c(3), 2} \xi+b_{h c(3), 3}=0
$$

where the $b_{h c(3), k}, k=1,2,3$ were listed in 25 


\section{Square Lattice, $\mathbf{P B C}_{y}$}

We first consider a strip of the square lattice with $\mathrm{PBC}_{y}$ and transverse cross sections forming triangles. Depending on one's labelling conventions, this corresponds to $L_{y}=3$

or $L_{y}=4$, where in the latter case, one interprets the periodic boundary conditions as identifying the top and bottom vertices for each value of $x$. We calculate

$$
W\left(s q\left(L_{y}=3\right), P B C_{y}, q\right)=\left(q^{3}-6 q^{2}+14 q-13\right)^{1 / 3}
$$

For the next larger size, i.e. transverse cross sections forming squares, corresponding to $L_{y}=4$ or $L_{y}=5$ in the respective labelling conventions described above, the $W$ function is given by 25]

$$
\begin{aligned}
& W\left(s q\left(L_{y}=4\right), P B C_{y}, q=2^{-1 / 4}\left[\left(q^{4}-8 q^{3}+29 q^{2}-55 q+46\right)\right.\right. \\
& \left.\quad+\left[q^{8}-16 q^{7}+118 q^{6}-526 q^{5}+1569 q^{4}-3250 q^{3}+4617 q^{2}-4136 q+1776\right]^{1 / 2}\right]^{1 / 4}
\end{aligned}
$$

\section{E. Triangular Lattice, $\mathbf{P B C}_{y}$}

We next consider a strip of the triangular lattice with $\mathrm{PBC}_{y}$, represented as a square lattice with additional diagonal bonds from, say, the upper left to lower right vertices of each square. For the case where the transverse cross sections form triangles, corresponding to $L_{y}=3$ or $L_{y}=4$ in the above labelling conventions, we calculate

$$
W\left(t\left(L_{y}=3\right), P B C_{y}, q\right)=\left(q^{3}-9 q^{2}+29 q-32\right)^{1 / 3}
$$

For the next larger size, with transverse cross sections forming squares, $W$ is [25]

$$
\begin{aligned}
& \left.W\left(t\left(L_{y}=4\right), F B C_{y}\right], q\right)=2^{-1 / 4}(q-3)^{1 / 4}\left[\left(q^{3}-9 q^{2}+33 q-48\right)\right. \\
& \left.\quad+(q-4)\left[q^{4}-10 q^{3}+43 q^{2}-106 q+129\right]^{1 / 2}\right]^{1 / 4}
\end{aligned}
$$


[1] L. Pauling, The Nature of the Chemical Bond (Cornell Univ. Press, Ithaca, 1960), p. 466.

[2] E. H. Lieb and F. Y. Wu, in C. Domb and M. S. Green, eds., Phase Transitions and Critical Phenomena (Academic Press, New York, 1972) v. 1, p. 331.

[3] M. Aizenman and E. H. Lieb, J. Stat. Phys. 24, 279 (1981).

[4] Y. Chow and F. Y. Wu, Phys. Rev. B36, 285 (1987); Y. Chow, Discrete Math, 66 (1987) $51-58$.

[5] R. B. Potts, Proc. Camb. Phil. Soc. 48 (1952) 106.

[6] F. Y. Wu, Rev. Mod. Phys. 54, 235 (1982); errata, ibid. 55, 315 (1983).

[7] R. C. Read, J. Combin. Theory 4, 52 (1968); R. C. Read and W. T. Tutte, "Chromatic Polynomials", in Selected Topics in Graph Theory, 3, eds. L. W. Beineke and R. J. Wilson (Academic Press, New York, 1988.)

[8] E. H. Lieb, Phys. Rev. 162, 162 (1967).

[9] R. J. Baxter, J. Phys. A 20, 5241 (1987).

[10] J. F. Nagle, J. Combin. Theory 10 (1971) 42; G. A. Baker, Jr., J. Combin. Theory 10 (1971) 217.

[11] D. Kim and I. G. Enting, J. Combin. Theory, B 26, 327 (1979).

[12] R. Shrock and S.-H. Tsai, Phys. Rev. E55, 6791 (1997).

[13] R. Shrock and S.-H. Tsai, Phys. Rev. E56, 2733 (1997).

[14] R. Shrock and S.-H. Tsai, Phys. Rev. E56,, 4111 (1997); S.-H. Tsai, Phys. Rev. E57, 2686 
(1998).

[15] N. L. Biggs, Bull. London Math. Soc. 9, 54 (1977).

[16] X. Chen and C. Y. Pan, Int. J. Mod. Phys. B1, 111 (1987); C. Y. Pan and X. Chen, ibid. B2, 1503 (1988).

[17] J.-S. Wang, R. H. Swendsen, and R. Kotecký, Phys. Rev. B 42, 2465 (1990).

[18] R. Shrock and S.-H. Tsai, J. Phys. A 30, 495 (1997).

[19] N. L. Biggs, R. M. Damerell, and D. A. Sands, J. Combin. Theory B 12, 123 (1972).

[20] S. Beraha, J. Kahane, and N. Weiss, J. Combin. Theory B 28, 52 (1980).

[21] R. C. Read and G. F. Royle, in Graph Theory, Combinatorics, and Applications (Wiley, New York, 1991), vol. 2, p. 1009.

[22] R. Shrock and S.-H. Tsai, Phys. Rev. E55, 5165 (1997).

[23] R. Shrock and S.-H. Tsai, Phys. Rev. E56, 1342 (1997).

[24] R. Shrock and S.-H. Tsai, Phys. Rev. E56, 3935 (1997); J. Phys. A, in press; ITP-SB-98-17.

[25] M. Roček, R. Shrock, and S.-H. Tsai, Physica A252, 505 (1998); Physica A259, 367 (1998).

[26] R. Shrock and S.-H. Tsai, Physica A259, 315 (1998).

[27] A. E. Ferdinand and M. E. Fisher, Phys. Rev. 185, 832 (1969).

[28] A. A. Belavin, A. M. Polyakov, and A. B. Migdal, Nucl. Phys. B241, 333 (1984); D. Friedan, Z. Ziu, and S. Shenker, Phys. Rev. Lett. 52, 1575 (1984); C. Itzykson, H. Saleur, and J.B. Zuber, Conformal Invariance and Applications to Statistical Mechanics (World Scientific, Singapore, 1988); J. Cardy, in C. Domb and J. L. Lebowitz, eds., Phase Transitions and Critical Phenomena (Academic Press, New York, 1987), vol. 11, p. 55. 
[29] J. Kondev and C. Henley, Nucl. Phys. B464, 540 (1996).

[30] H. Park and M. den Nijs, Phys. Rev. B38, 565 (1988); J. Phys. A 22, 3663 (1989); H. Park and M. Widom, Phys. Rev. Lett. 63, 1193 (1989)

[31] J. L. Cardy, J. Phys. A 17, L385, L961 (1984); H. W. J. Blöte, and M. P. Nightingale, Phys. Rev. Lett. 56, 742 (1986); I. Affleck, Phys. Rev. Lett. 56, 746 (1986). 